\title{
Los efectos de la financiación pública en el desempeño innovador y económico empresarial
}

\author{
The Effects of Public Financing on Innovative and Economic Business Performance
}

\author{
Fanny Cabrera-Barbecho $^{1}$ (D), Cristina Chapa-Zumba ${ }^{2}$ iD, Juan Sarmiento-Jara ${ }^{3}$ (D), Víctor \\ Aguilar-Feijó ${ }^{3}$ \\ ${ }^{1}$ Economista estudiante de la Maestría de Economía de la Facultad de Ciencias Económicas y Administrativas, \\ Universidad de Cuenca. \\ ${ }^{2}$ Economista por la Facultad de Ciencias Económicas y Administrativas, Universidad de Cuenca. \\ ${ }^{3}$ Profesor investigador de la Facultad de Ciencias Económicas y Administrativas, Universidad de Cuenca. \\ Autor para correspondencia: victor.aguilar@ucuenca.edu.ec \\ Fecha de recepción: 18 de marzo de 2020 - Fecha de aceptación: 6 de abril de 2020
}

\section{RESUMEN}

La existencia de fallas de mercado induce a que la inversión privada en actividades de innovación sea menor a la socialmente óptima. De ahí que la intervención del estado puede ser fundamental para promover actividades de innovación tendientes a corregir este problema. El presente artículo, mediante la estimación de un sistema de ecuaciones en su forma reducida, evalúa si la financiación pública estimula la inversión en innovación y mejora el desempeño innovador y económico empresarial en el Ecuador, utilizando la Encuesta de Actividades de Innovación del Instituto Nacional de Estadística y Censos, versiones 2009-2011/2012-2014. Se encontró que el apoyo público incrementa la inversión en innovación, promoviendo fundamentalmente la innovación exógena. En cuanto a los efectos sobre la capacidad de innovar en producto o proceso y a los efectos sobre la productividad, se encontraron resultados que difieren de acuerdo al periodo de análisis y al sector económico.

Palabras clave: Financiación, inversión, innovación, producto, proceso.

\begin{abstract}
The existence of market failures induces that private investment in innovation activities is less than socially optimal. Hence, state intervention can be essential to promote innovation activities aimed at correcting this problem. This article, by estimating a system of equations in its reduced form, evaluates whether public financing stimulates investment in innovation and improves innovative and economic business performance in Ecuador, using the Innovation Activities Survey of the National Institute of Statistics and Censuses, versions 2009-2011/2012-2014. Public support was found to increase investment in innovation, fundamentally promoting exogenous innovation. Regarding the effects on the ability to innovate in product or process and the effects on productivity, results were found different according to the period of analysis and the economic sector.
\end{abstract}

Keywords: Financing, investment, innovation, product, process

\section{INTRODUCCIÓN}

El Manual de Oslo concibe a la innovación como la introducción de un nuevo o significativamente mejorado, producto, proceso, método de comercialización o un nuevo método de organización (OCDE-Eurosat, 2005), y es considerada como un factor estratégico para mejorar la productividad de las empresas. Dada la importancia de realizar actividades de innovación, es relevante preguntarse por qué no todas las empresas invierten en ella. Jiménez (2008) indica que lo que hace posible a una firma llevar a cabo actividades innovadoras es una financiación adecuada; pero no todas las empresas cuentan con los fondos para financiar actividades innovadoras, debido a que su obtención se dificulta por las fallas de mercado (Aboal \& Garda, 2015; Aghion, David, \& Foray, 2009; Greenhalgh \& Rogers, 2009; Nooteboom \& Stam, 2008)

Entre estas fallas está el problema de apropiación: al ser la innovación un bien no rival y no excluyente, genera externalidades positivas, y como consecuencia es posible que las empresas que realizan estas actividades no puedan apropiarse por completo del rendimiento que genera sus inversiones en innovación (Greenhalgh \& Rogers, 2009). No obstante, Aboal \& Garda (2015) argumentan que a pesar de que la firma utilice algún medio de protección intelectual, existen otros tipos de problemas, como la incertidumbre de si los resultados obtenidos son los esperados, o si invertir en actividades de innovación efectivamente permite lograr innovaciones en productos o 
procesos. Un tercer problema es la asimetría de la información, que conlleva a escenarios de riesgo moral y selección adversa ${ }^{1}$ (Crespi et al., 2015; Hall \& Lerner, 2009). Finalmente, la conjugación de las anteriores causas conduce a una falla adicional, denominada falla de coordinación, en el sentido de que existe un desincentivo a que innovador e inversionista cooperen, debido a la desconfianza (Crespi et al., 2015).

Por estos motivos, la inversión en innovación privada es menor a la inversión óptima social (Aboal \& Garda, 2015; Greenhalgh \& Rogers, 2009; López, 2009; Nooteboom \& Stam, 2008), justificando con ello la intervención del gobierno en la estimulación de la misma para lograr eficiencia en términos económicos y sociales (Bukstein \& Usher, 2016; Vila, Ferro, \& Guisado, 2009). Así, Barona et al. (2015) indican que en un país donde no se encuentran desarrollados los mercados de acciones y capital el apoyo público en innovación se vuelve aún más importante. Sin embargo, dado que los recursos públicos son escasos, resulta de vital importancia determinar si efectivamente las políticas de financiación proporcionan los efectos esperados (Guaipatin \& Schwartz, 2014; López, 2009).

En el caso particular del Ecuador, aun cuando autores como Guaipatin \& Schwartz (2014) estiman que el retorno social de la inversión en Investigación y Desarrollo (I\&D) se encuentra en alrededor del $47 \%$-porcentaje mayor al retorno social de invertir en capital físico que es del $12 \%$ el país continúa aún muy rezagado en esta área; de acuerdo a la Organización Mundial de Propiedad Intelectual (OMPI), en 2019 ocupó el puesto 99 entre 129 economías a nivel mundial en el ranking del Índice Global de Innovación y el puesto 14 entre 19 países de Latinoamérica. En términos de gasto en investigación y desarrollo (I\&D) también mantiene un rezago con respecto a la región; entre 2009 y 2014 se gastó en promedio $0.38 \%$ del PIB en este rubro, menor al promedio de América Latina que fue $0.74 \%$ (Red de Indicadores de Ciencia y Tecnología Iberoamericana e Interamericana RICYT, 2016).

La evidencia empírica del impacto que tiene la financiación pública en Ecuador es escasa, debido en parte a que la primera encuesta exhaustiva sobre actividades de innovación se publica recién en el año 2013. Además, como indican Guaipatin \& Schwartz (2014), influyen otros factores tales como el marco regulatorio y clima de negocios, las restricciones crediticias y la vulnerabilidad económica y política que han caracterizado al país, condiciones que desincentivan a los agentes a realizar inversión en actividades de innovación y no generan suficiente interés de evaluar la misma.

Con el fin de aportar evidencia empírica sobre el tema, esta investigación tiene como objetivo evaluar si la financiación pública ha tenido un efecto sobre la inversión en innovación en productos o procesos, así como en el desempeño innovador y económico de las empresas privadas del sector manufactura y servicios del Ecuador durante los periodos 2009-2011 y 2012-2014.

\subsection{Revisión de la literatura}

Siguiendo a Huergo, Trenado \& Ubierna (2015), la

${ }^{1}$ Según Greenhalgh y Rogers (2009), el problema de asimetría de la información hace referencia a que el agente que innova intervención pública en el financiamiento de la innovación se justifica para corregir el desincentivo de las fallas de mercado antes mencionadas, sin embargo, es probable que esta intervención no tenga los efectos esperados, lo que hace necesario evaluar la efectividad de dicha intervención (Crespi et al., 2015; López, 2009). El efecto deseado es que la financiación pública estimule el gasto en innovación en las empresas; al respecto, López (2009) menciona que aquello es posible cuando la firma beneficiada se enfrenta a dificultades de financiamiento, haciendo que las empresas utilicen los recursos públicos para financiar estas actividades en lugar de remplazarlos. Sin embargo, es posible que en lugar de ello solamente se sustituyan los recursos privados con públicos, produciendo un efecto exclusión o sustitución, denominado crowding out.

A pesar de este posible escenario, autores como Aerts \& Schmidt (2008), Carboni (2011), Czarnitzki \& LopesBento (2014), González \& Pazó (2008), Hud \& Hussinger (2015) concluyen que existe evidencia de que el financiamiento público de la innovación genera efectos positivos sobre el esfuerzo innovador y/o gastos en I\&D en empresas europeas, descartando un efecto desplazamiento. Este resultado es avalado en mucha de la literatura respecto a los países emergentes y en vías de desarrollo de América Latina (Bukstein \& Usher, 2016; Crespi, Solís, \& Tacsir, 2011). Dai \& Cheng (2015) advierten que en realidad no se evidencia un efecto único (adicionalidad o crowding out), sino que estos difieren dependiendo del monto del subsidio. En este sentido, los autores encuentran para empresas manufactureras de China una relación de $\mathrm{U}$ invertida entre la inversión de I\&D privada y los subsidios públicos, lo que a su vez significa que existe un intervalo óptimo de subsidio donde se evidencia efectos de adicionalidad; sobrepasar el intervalo significa un efecto desplazamiento que dependerá del nivel de subsidio. No obstante, una de las limitaciones más comunes de los autores es la falta de información para realizar análisis de efectos diferenciados del apoyo público (Czarnitzki \& Lopes-Bento, 2014).

Autores como Arza \& López (2010), Zúñiga \& Crespi (2013) indican que la decisión de realizar determinado tipo de actividades de innovación tendrá efectos distintos en el desempeño innovador y económico de la firma; de esta manera, dividen las actividades de innovación en dos: endógena (In-house) y exógena (External Source). La primera se desarrolla al interior de la empresa y consta de I\&D interna, actividades de ingeniería y diseño industrial; las actividades de innovación exógena están compuestas por I\&D externa, adquisición de bienes de capital, adquisición de tecnologías de la información y la comunicación, transferencias de tecnología y consultorías, diseño organizacional gestión y capacitación (INEC, 2011).

En este contexto, se espera que ambos tipos de actividades generen mayor productividad; no obstante, realizar actividades internas es preferible (Jaramillo et al., 2001), ya que crea capacidades propias para la economía en conjunto con una reducción de dependencia de tecnología externa. De acuerdo a Lasarga, Rosich, \& Rueda (2015) es probable que en una economía en desarrollo la financiación pública incentive a realizar actividades de

tiene mayor información sobre los retornos de la innovación en comparación con el agente que invierte. 
innovación exógenas, debido a la necesidad de las firmas de absorber capacidades antes de crear innovaciones (Howell, 2017).

Según Bukstein \& Usher (2016) no solo se debe estudiar la existencia o no de efectos de desplazamiento de fondos públicos por privados, ya que invertir en innovación no necesariamente significa que el resultado del mismo tenga éxito. Adicionalmente, se debe realizar un análisis a mediano plazo que evalúe los efectos de la financiación pública sobre la capacidad de introducir al mercado un nuevo o significativamente mejorado producto $\mathrm{y} / \mathrm{o}$ proceso.

Entre la literatura que estudia esta dimensión se tiene además a Herrera (2012) para empresas españolas, Hewitt-Dundas \& Roper (2010) para firmas manufactureras irlandesas, Szczygielski et al. (2016) para empresas de Turquía y Polonia, quienes encuentran que el apoyo público tiene efectos positivos sobre la introducción de innovaciones de producto o proceso, o sobre las ventas de productos nuevos o mejorados. Sin embargo, Radicic \& Pugh (2015) para medianas y pequeñas empresas europeas evidencian efectos positivos sobre la propensión de solicitud de patentes, pero no sobre ventas innovadoras; Czarnitzki \& Lopes-Bento (2014) encuentran que las empresas financiadas patentarán más en el período $t+1$ con respecto a las no financiadas, pero evidencian efectos inconclusos sobre ventas con novedades en el mercado. Benavente, Crespi \& Maffioli (2007) señalan que no existe evidencia de efectos significativos para la creación de nuevos productos en empresas chilenas, pero es posible que exista efectos inconclusos debido a que dichos efectos se observan en un lapso de tiempo mayor que el analizado.

A más de los efectos mencionados Bukstein \& Usher (2016), López (2009) argumentan que la innovación no es el objetivo final, sino que es un medio por el que las empresas alcanzan una mayor rentabilidad y desempeño económico, efecto que se visualiza en el largo plazo. En este contexto, es relevante evaluar si efectivamente la financiación pública conlleva a un mayor desempeño de las empresas beneficiadas en comparación con las no beneficiadas. Varios estudios que analizan los efectos del apoyo público sobre el desempeño económico de las empresas no evidencian que el apoyo gubernamental aumenta la productividad de las firmas beneficiadas (Bukstein \& Usher, 2016; Chudnovsky et al., 2006; De Negri, Borges Lemos, \& De Negri, 2006). Particularmente para América Latina, Benavente et al. (2007) encuentran que las empresas participantes del fondo de desarrollo tecnológico mejoran el crecimiento de la empresa en términos de ventas, número de empleados $\mathrm{y}$ exportaciones, pero no se observa impactos significativos en su productividad.

Por el contrario, autores como Crespi et al. (2015) para firmas colombianas, Castro \& Jorrat (2013) para empresas de servicios argentinos, demuestran que la ayuda gubernamental para las actividades de I\&D e innovación contribuye a un mejor rendimiento de las empresas. En particular, Castro \& Jorrat (2013) encuentran efectos positivos de los beneficios fiscales, mas no de los subsidios, sobre la productividad de las Pymes del sector servicios de software informáticos en Argentina. De manera que la evidencia en este aspecto no es concluyente, fundamentalmente porque se requeriría de un tiempo más extenso para observar el impacto del apoyo público sobre la productividad.
Autores como Aboal \& Garda (2015), Audretsch, Coad, \& Segarra (2014), Lee (2011), enfatizan que el impacto de la financiación pública sobre la innovación es distinto, dependiendo de las características de cada empresa, industria y país. Por tanto, analizar la efectividad de la misma a nivel agregado $y$ hacer conclusiones generalizadas puede no aplicarse a todas las industrias (Lee, 2011). Es posible que existan efectos heterogéneos del apoyo gubernamental sobre las firmas de diferentes sectores económicos. Por ejemplo, se considera que el impacto puede ser mayor en el sector de manufactura debido a sus fuertes encadenamientos productivos con otros sectores. Por otro lado, el sector servicios representa más del $70 \%$ del PIB en las economías desarrolladas y más del $60 \%$ en las economías en vías al desarrollo (Aboal \& Garda, 2015); según Cimoli (2010), las innovaciones de este sector tienen efectos en el resto de la economía, tanto en términos de eficiencia como de competitividad y complementariedad. No obstante, los costos de transacción para innovar en el sector servicios son más altos en comparación con el de manufactura, debido a la intangibilidad de los servicios; esta es la razón por la que es más complejo proteger innovaciones en este sector y por ende la incorporación de la inversión al rendimiento es más dificultosa.

Dado lo descrito y la posición del país en este contexto, nuestras hipótesis de investigación son: (1) la financiación pública estimula la intensidad de innovación; (2) en economías en desarrollo el apoyo público incentiva realizar actividades de innovación exógena más que endógena; (3) el apoyo gubernamental aumenta la capacidad de introducir innovaciones de producto y/o proceso; (4) las firmas beneficiadas presentan mayor productividad que las no beneficiadas; (5) finalmente, el impacto de la política difiere dependiendo del sector manufactura o servicios a la que pertenece la firma.

\section{MATERIALES Y MÉTODOS}

Los datos utilizados provienen de la Encuesta Nacional de Innovación (ENAI), rondas 2009-2011 y 2012-2014, realizada por el Instituto Nacional de Estadísticas y Censos (INEC), en conjunto con la Secretaría Nacional de Educación superior, Ciencia y Tecnología (SENESCYT), a las empresas pertenecientes a los sectores de extracción de minas y canteras, industrias manufactureras, comercio y servicios, de acuerdo con la Clasificación Industrial Internacional Uniforme (CIIU 4.0). Así, para el trienio 2009-2011, se recolectó información de 2,815 empresas, mientras que para el trienio 2012-2014 de 6,275 empresas. De acuerdo con los objetivos de este estudio, la muestra utilizada se limita a las empresas privadas de manufactura y servicios, por lo que la muestra final para el periodo 2009-2011 fue de 2,485 empresas, 1,178 (47\%) de manufactura y $1,307(53 \%)$ de servicios; mientras que para el periodo 2012-2014 el total de observaciones incluidas en el análisis fue de 4,044 empresas, de las cuales $1,610(40 \%)$ pertenecen al sector manufactura y $2,434(60 \%)$ al sector servicios.

La muestra final de empresas incluye tanto aquellas que realizaron algún tipo de innovación durante el periodo de análisis, como aquellas que no lo hicieron. Esta decisión trae consigo algunas ventajas, pero también algunos problemas en la muestra y en la estimación. Por un lado, 
la muestra con la que se trabaja es mayor que aquella resultante si se hubiera considerado en el análisis únicamente las empresas que tuvieron un gasto positivo en innovación. Por otro lado, el número de variables explicativas que es posible utilizar se limita, ya que algunas de ellas solamente se registraron para las empresas que realizaron algún tipo de innovación. Además, al momento de modelar el gasto en innovación se presenta un fuerte problema de censura, debido a la existencia de observaciones con valor igual a cero.

A partir del trabajo seminal de Crépon, Duguet, \& Mariesse (1998), la literatura empírica ha desarrollado varios estudios que analizan el proceso de innovación aplicando este enfoque conocido como CDM. De manera particular, varias investigaciones utilizan el modelo CDM para analizar el efecto específico de uno de los determinantes de la innovación, entre ellos, Czarnitzki \& Delanote (2017) aplican un enfoque CDM ampliado para analizar el efecto de los subsidios públicos a la inversión en I+D. En este estudio, la metodología utilizada plantea la estimación de un sistema de ecuaciones en su forma reducida basados en el modelo CDM, buscando modelar todo el proceso involucrado en la innovación, que va desde la decisión de la empresa sobre el monto a erogar en actividades de innovación, hasta el efecto de la innovación en el desempeño de la productividad laboral. Se incluyen tres relaciones causales.

La primera relaciona la intensidad de la innovación, medida por el logaritmo del gasto en innovación, con sus determinantes; esta relación puede ser representada mediante la siguiente ecuación:

$$
y_{i}=\boldsymbol{x}_{1 i}^{\prime} \boldsymbol{\beta}_{1}+\alpha_{1} F P_{i}+u_{i}
$$

Donde $y_{i}$ representa el logaritmo del gasto en innovación realizado por la empresai-ésima, $\boldsymbol{x}_{l i}$ es un vector de controles determinantes del gasto en innovación, $F P_{i}$ representa al tratamiento y toma el valor de 1 si la empresa recibió algún tipo de financiamiento público para realizar actividades de innovación y 0 en caso contrario. Finalmente, $u_{i}$ es un término aleatorio, que se asume ruido blanco.

La segunda relación da cuenta del efecto del gasto en innovación y la financiación pública en resultados tangibles de la innovación, tales como nuevos productos o procesos. Para ello se plantean dos ecuaciones; la primera, está representada por la siguiente expresión:

$$
p d_{i}^{*}=\alpha_{y d} \hat{y}_{i}++\alpha_{3} F P_{i}+\boldsymbol{x}_{2 i}^{\prime} \boldsymbol{\beta}_{2}+u_{2 i}
$$

Donde $p d_{i}^{*}$ es una variable latente, tal que observamos $p d_{i}=1$ si la empresa logró generar algún producto nuevo, y $p d_{i}=0$, si no lo hizo. La variable $\hat{y}_{i}$ es el logaritmo del gasto en innovación estimado en la ecuación previa, mientras que $\boldsymbol{x}_{2 i}$ representa el vector de controles. En la práctica $\boldsymbol{x}_{2}$ puede ser igual a $\boldsymbol{x}_{1}$. Una segunda ecuación, está dada por:

$$
p r_{i}^{*}=\alpha_{y r} \hat{y}_{i}+\alpha_{3} F P_{i}+\boldsymbol{x}_{3}^{\prime} \boldsymbol{\beta}_{3}+u_{3 i}
$$

Donde $p r_{i}^{*}$ es una variable latente, tal que observamos $p r_{i}=1$ si la empresa logró generar algún proceso nuevo, y $p r_{i}=0$, si no lo hizo. Las demás variables se explican por sí mismas.
La última relación se establece entre la productividad laboral con los productos derivados de la innovación y con el financiamiento público, de la siguiente manera:

$$
q_{i}=\alpha_{d ; r}\left(p d_{i}^{*} ; p r_{i}^{*}\right)+\alpha_{4} F P_{i}+\boldsymbol{x}_{4}^{\prime} \boldsymbol{\beta}_{4}+u_{4 i}
$$

Donde, asumiendo una función de producción tipo CobbDouglas, $q_{i}$ es el logaritmo productividad (logaritmo de las ventas por trabajador), mientras que el vector $\boldsymbol{x}_{4}$ incluye variables como el logaritmo del número de empleados, el logaritmo del capital físico por empleado, entre otras.

\subsection{Endogeneidad, censura y otros problemas econométricos}

Existen cuatro problemas econométricos que deben ser enfrentados. En primer lugar, surge el problema de sesgo de selección, debido a que el financiamiento público a las empresas no se realiza de manera aleatoria. Los organismos estatales al otorgar apoyo público utilizan algún tipo de proceso de selección, en la que se eligen a las empresas de acuerdo a ciertas características que afectan a las variables de resultado, es decir, características que influyen en el éxito del programa y por tanto no habría independencia entre el tratamiento y el resultado (Aboal \& Garda, 2015; López, 2009). Como consecuencia no se debe estimar de forma directa la ecuación (1), ya que no existe aleatoriedad en la selección para el tratamiento.

Existen varias metodologías econométricas que toman en cuenta este problema, entre las que se encuentran los métodos de emparejamiento, diferencias en diferencias, regresión discontinua y variables instrumentales (López, 2009; Wooldridge, 2002). Debido a los fuertes supuestos del método de emparejamiento, al hecho de que la metodología de diferencia en diferencias necesita información tanto antes como después del tratamiento, y que no se cuenta con un índice de elegibilidad continuo para aplicar el método de regresión discontinua, en la presente investigación se aplica el método de variables instrumentales (VI), utilizado por autores como HewittDundas \& Roper (2010), Huergo et al. (2015). Esta metodología se aplica mediante dos etapas; en la primera se estima una ecuación de selección, para determinar la probabilidad de recibir el tratamiento a través de un modelo probit (Cerulli, 2011; Wooldridge, 2002). En términos formales se tiene:

$$
F P_{i}(\boldsymbol{x})=p(F P=1 \mid \boldsymbol{x})
$$

Donde $F P_{i}(\boldsymbol{x})$ toma el valor de 1 si la empresa recibe apoyo financiero del gobierno y el valor de 0 en caso contrario. En la segunda etapa se requiere de uno o más instrumentos que sean relevantes y exógenos con el término de error (Wooldridge, 2009, p.508); para ello se utiliza la probabilidad estimada $\widehat{F P}_{i}$ de la ecuación (5) como instrumento para la ecuación (1):

$$
y_{i}=\boldsymbol{x}_{1 i}^{\prime} \boldsymbol{\beta}_{1}+\alpha_{1} \widehat{F P}_{i}+u_{i}
$$

Por lo tanto, si $\widehat{F P}_{i}$ es un instrumento válido y no está correlacionado con el error, $\hat{\alpha}_{1}$ es una estimación válida para el efecto medio del tratamiento (ATE) (Wooldridge, 
$2002)^{2}$.

El segundo problema econométrico a enfrentar es el problema de la endogeneidad que se presenta en las ecuaciones (2) y (3) debido a que la variable dependiente de la primera relación es endógena en las ecuaciones de los productos de la innovación; por tanto, es probable que los errores de estas ecuaciones estén correlacionados. Para solventar este problema se utiliza, tal como se aprecia en las ecuaciones (2) y (3), el valor estimado de la variable dependiente correspondiente a la primera ecuación, es decir $\hat{y}_{i}$.

Adicionalmente, tenemos el problema de la censura en la ecuación (6). Es factible asumir que un porcentaje importante de empresas toman una solución de esquina, es decir no realizan gastos en innovación ya que presentan utilidades marginales negativas de invertir en innovación $\mathrm{y}$, como consecuencia, prefieren no hacerlo; mientras que para valores positivos en esta variable de resultado existe una distribución continua, característica necesaria para aplicar un modelo tobit, con variable dependiente limitada con censura a la izquierda en cero. Formalmente, se expresa $y_{i}$ en términos de una variable latente no observada, $y_{i}^{*}$ :

$$
y_{i}^{*}=\boldsymbol{x}_{1 i}^{\prime} \boldsymbol{\beta}_{1}+\alpha_{1} \widehat{F P}_{i}+u_{i}
$$

Con $u_{i} \sim N\left(0 ; \sigma^{2}\right)$. Además $y_{i}=y_{i}^{*}$ si $y_{i}^{*}>0, y_{i}=0 \mathrm{si}$ $y_{i}^{*} \leq 0$.

Finalmente, el último aspecto econométrico a considerar es el de la posible correlación entre los errores de las ecuaciones de resultado de la innovación, es decir la posibilidad de que $\operatorname{Cov}\left(u_{2 i} ; u_{3 i}\right) \neq 0$. Para dar cuenta de esta posibilidad, se estima el efecto promedio del tratamiento sobre la probabilidad de innovar en producto y/o proceso mediante un modelo probit bivariante.

\subsection{Variables y relaciones esperadas}

Las variables de resultado que se emplean para medir la efectividad del tratamiento se utilizan de acuerdo a las especificaciones de Aboal \& Garda (2015), Bukstein \& Usher (2016), Herrera (2012), Lasarga et al. (2015). Con respecto al impacto del financiamiento público sobre el monto de inversión en innovación (intensidad de innovación), se emplean las siguientes variables: Inversión total en innovación e inversión de fuente privada, esta última para capturar un posible efecto de crowding out; la primera se mide como el logaritmo del gasto en I\&D mas esfuerzos innovativos en producto y proceso, por empleado; la segunda como el logaritmo del gasto en I\&D mas esfuerzos innovativos en producto y proceso menos el porcentaje de financiación pública en innovación, por empleado. Inversión en actividades relacionadas a la innovación endógena (in house) e inversión en actividades de innovación exógena (external source), para capturar el efecto del financiamiento público en la intensidad de la innovación, de acuerdo al tipo de actividades de innovación; se mide como el logaritmo del gasto en innovación endógena y exógena por empleado, respectivamente.

\footnotetext{
2 Cabe acotar que ATE representa el efecto medio de la financiación pública en innovación sobre la variable de resultado siempre y cuando los efectos del apoyo sean homogéneos (iguales en magnitud para todas las firmas).
}

Con respecto a los efectos sobre la capacidad de innovar, se incluye las variables innova en productos e innova en procesos, que toman el valor de 1 si la empresa logró introducir nuevos productos (o procesos) significativamente mejorados, o 0 en caso contrario, durante el periodo de análisis. Finalmente, para determinar los efectos en el desempeño de la empresa, se utiliza la variable productividad laboral medida a través del logaritmo de las ventas por empleado.

Variables explicativas: la política a ser evaluada es la financiación pública ${ }^{3}$; así, la variable de tratamiento es binaria, codificada como 1 si la empresa es beneficiaria, o como 0 en caso contrario. Siguiendo la metodología del apartado anterior, las variables de control corresponden a características observables de las empresas y de su entorno que influyen en la probabilidad de selección para otorgar financiamiento público, en la intensidad de la innovación, en la probabilidad de innovar en productos y/o procesos y en la productividad laboral. A continuación, se explica brevemente los controles utilizados en las distintas ecuaciones a estimar. El detalle sobre el signo esperado y período en el que se toma cada variable se encuentra en el Anexo 1.

Factores que afectan la probabilidad de recibir financiamiento público, la intensidad y resultados de la innovación: según Hall (2010) las empresas pequeñas y jóvenes tienen mayor probabilidad de fracasar en proyectos de innovación, dado que se enfrentan a un mayor costo de capital y problemas de financiamiento frente a firmas grandes y con mayor experiencia. En caso de que el gobierno aplique instrumentos de política para impulsar empresas con dificultades de financiamiento, se espera una relación negativa del tamaño y edad con respecto a la probabilidad de recibir financiación pública; por otro lado, cuando los organismos públicos tengan estrategias de "picking the winner", se espera una relación directa, es decir, que con el fin de reducir la probabilidad de fracaso en proyectos de innovación se favorece aquellas firmas con mayor capacidad de innovar (Aerts \& Schmidt, 2008; Dai \& Cheng, 2015).

Una empresa que pertenece a un grupo empresarial puede tener menor dificultad para innovar y mayor capacidad de generar un efecto spillover, dado el vínculo que tiene con otras empresas de la casa matriz (Klette, Møen, \& Griliches, 2000); para estas empresas se espera una menor probabilidad de recibir financiamiento público cuando los organismos estatales desean impulsar aquellas firmas con mayor dificultar de innovar (Clausen, 2009; Dai \& Cheng, 2015; Szczygielski et al., 2016). Un comportamiento similar se espera para empresas que cuentan con capital de origen extranjero.

En cuanto a la capacidad de las firmas para realizar actividades de innovación, esta sería mayor cuanto mayor sea la intensidad en capital fijo (Dai \& Cheng, 2015; Guan \& Pang, 2017); sin embargo, evidenciando una posición contraria, Czarnitzki \& Lopes-Bento (2014) mencionan que una de las razones para que una firma cuente con alta intensidad de capitales es porque se enfrenta a mayores barreras de entrada, y por tanto puede ser menos propensas a innovar. Acs \& Audretsch (1987) evidencian que las firmas pequeñas con alta intensidad de capital no son

\footnotetext{
3 La financiación pública hace referencia a si la firma obtuvo fondos de organismos públicos que fomentan la innovación en productos o procesos; no se especifica si se trata de un préstamo o de un subsidio.
} 
incentivadas a realizar actividades de innovación. Una vez más el signo esperado para la probabilidad de recibir financiamiento público depende de la estrategia de los organismos estatales.

Siguiendo a Herrera (2012), si una firma se sitúa en una región geográfica de alta concentración industrial es posible que presente mayores oportunidades de desarrollo, no solo para conseguir recursos sino para obtener resultados de sus innovaciones. Entonces, dependiendo de la estrategia del estado, éste puede por un lado apoyar a aquellas empresas situadas en regiones con menos capacidad para innovar, o proporcionar mayor apoyo a las empresas ubicadas en regiones económicamente más desarrolladas. Además, las firmas pertenecientes a algún sector considerado estratégico del país podrían tener una mayor propensión a recibir financiamiento público.

Las empresas con más experiencia y capacidades tendrán mayores posibilidades de innovar, por lo que se espera una relación directa con respecto a variables como la intensidad de innovación previa, la existencia de un departamento en I\&D y la experiencia en la participación en programas públicos de apoyo a la innovación.

Las empresas que exportan se enfrentan a mercados más competitivos, por lo que la innovación puede ser una estrategia importante para generar mayor valor a sus firmas y sobrevivir en el mercado (Aerts \& Schmidt, 2008); se espera que las empresas exportadoras tengan mayor probabilidad de recibir apoyo cuando los gobiernos están dispuestos a financiar firmas que se enfrentan a mayor competitividad en el mercado para promover la demanda comercial (Clausen, 2009).

Se consideran además variables que den cuenta del acceso a otras fuentes de financiamiento para la innovación: recursos propios, banca y recursos del exterior. De esta manera, si una firma tiene un bajo porcentaje en estas fuentes para financiar actividades de innovación, presentarán mayor restricción financiera y por lo tanto mayor probabilidad de recibir recursos del estado (Huergo et al., 2015).

En cuanto al momento temporal de las variables, algunas de ellas se toman al comienzo del período analizado con el fin de evitar problema de endogeneidad (Gertler et al., 2009), entre las que tenemos: tamaño, capital fijo, gasto en actividades de innovación, competitividad y productividad. El resto de variables no presentan este tipo de problemas ya que están medidas para todo el periodo analizado.

\section{RESULTADOS}

En esta sección se analizan algunas características de las empresas estudiadas y su desempeño innovador, con el fin de determinar si existen diferencias significativas entre el grupo de tratados y no tratados. En la Tabla 1 se presenta el porcentaje de empresas que realizan actividades de innovación en producto o proceso, así como la proporción de firmas que reciben apoyo financiero público por sector.

Aproximadamente la mitad de las empresas consideradas realizaron algún tipo de actividades en innovación, aunque el porcentaje disminuye entre los dos periodos analizados. Por otro lado, la proporción de firmas que reciben financiamiento es bastante baja: menos del $3 \%$ en todos los casos. Se observa, además, una disminución en la proporción de firmas beneficiadas para el segundo periodo en el sector servicios, frente a un aumento en el sector manufacturero.

\section{Probabilidad de recibir financiamiento público}

El Anexo 2 presenta los efectos marginales asociados a la ecuación (5); las columnas 1-2 corresponden al total de empresas para los periodos 2009-2011 y 2012-2014, respectivamente. En términos generales se puede afirmar que una firma tiene mayor propensión a obtener apoyo público si ha realizado gastos en innovación en años previos, si ha participado de algún programa de apoyo a la innovación y si se enfrenta a dificultades financieras. A más de los factores mencionados, en particular para el periodo 2009-2011 se evidencia un efecto importante de la edad y de la pertenencia a un sector estratégico.

Al comparar entre sectores, vemos que para la industria manufacturera la importancia de contar con experiencia previa en actividades de innovación es mayor en comparación con el sector servicios. Además, las firmas manufactureras tienen menor probabilidad de recibir apoyo cuando cuentan con fondos para financiar sus actividades. Este resultado se explica porque las empresas de servicios enfrentan mayores problemas de apropiabilidad de los retornos de sus innovaciones, debido a las características intangibles de los servicios (Aboal \& Garda, 2015).

Tabla 1. Empresas privadas con actividades de innovación y apoyo financiero gubernamental: 2009-2014.

\begin{tabular}{lcccccc}
\hline \multirow{2}{*}{ Sector } & 1 & 2 & 3 & 4 & 5 & 6 \\
\cline { 2 - 7 } & \multicolumn{2}{c}{ \% de empresas } & $\begin{array}{c}\text { \% de empresas con actividades } \\
\text { de innovación en } \\
\text { producto/proceso }\end{array}$ & $\begin{array}{c}\text { \% de empresas con apoyo } \\
\text { financiero público }\end{array}$ \\
\cline { 2 - 7 } & $2009-2011$ & $2012-2014$ & $2009-2011$ & $2012-2014$ & $2009-2011$ & $2012-2014$ \\
\hline Manufactura & 47.4 & 39.8 & 60.95 & 58.30 & 1.78 & 2.80 \\
Servicios & 52.6 & 60.2 & 49.30 & 44.0 & 1.91 & 1.00 \\
Total & 100 & 100 & 54.81 & 49.70 & 1.85 & 1.70 \\
\hline
\end{tabular}

Fuente: elaboración propia sobre la base de datos de la Encuesta Nacional de Innovación (ENAI) de los periodos 2009-2011 y 2012-2014 del INEC. 
Tabla 2. Efecto medio de la financiación pública a nivel agregado en el periodo 2009-2014.

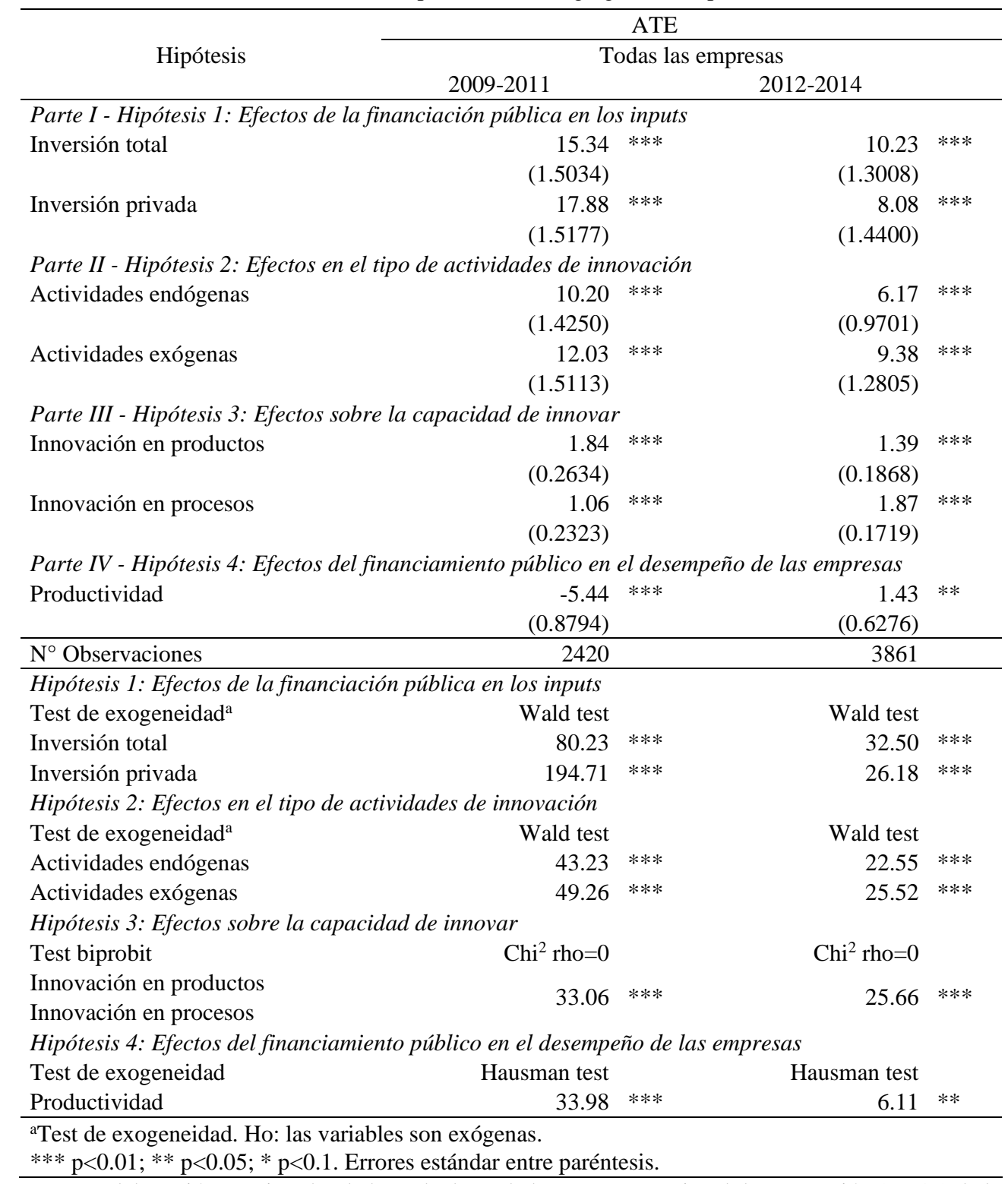

Fuente: elaboración propia sobre la base de datos de la Encuesta Nacional de Innovación (ENAI) de los periodos 2009-2011 y 2012-2014 del Instituto Nacional de Estadísticas y Censos (INEC).

Estos primeros hallazgos evidencian que los organismos estatales tienden a financiar a las firmas con experiencia previa en innovación y que tienen problemas de financiamiento, debido a que dichas firmas tienen mayor probabilidad de conseguir éxito en sus innovaciones. Este resultado es consistente con los hallazgos de Herrera (2012) y Huergo et al. (2015).

Efecto medio del tratamiento sobre las variables de resultados

La Tabla 2 muestra los efectos estimados del financiamiento público sobre la intensidad de la innovación, distinguiendo entre el gasto de innovación total y privado, así como entre el gasto de innovación en actividades endógena vs. exógenas; sobre la capacidad de innovar (innovación en productos/procesos) y sobre el rendimiento de las empresas (productividad laboral). Para validar la metodología de variables instrumentales se realiza el test de exogeneidad; se puede ver que se rechaza la hipótesis nula de que las variables del modelo sin instrumentos son exógenas $(p=0)$. En la misma tabla se valida el uso del modelo probit bivariante para modelar las ecuaciones de innovación en productos/proceso.

Un primer resultado denota que el financiamiento público aumenta la inversión en actividades de innovación, tanto cuando se considera el total de la inversión, como cuando se considera únicamente la inversión de fuentes privadas. Los signos obtenidos dan cuenta de un escenario donde no se rechaza la hipótesis de efectos positivos de fondos públicos o crowding in. A nivel sectorial (Anexo 3) no se reflejan diferencias en los signos obtenidos, pero si se ve un efecto heterogéneo en la magnitud del impacto; así, el efecto medio de la financiación pública para estimular la inversión en actividades de innovación es mayor en las empresas del sector manufactura.

Cuando no se considera el problema de endogeneidad se subestima el efecto de la financiación pública. Esto es 
consistente con la literatura empírica en el cual concluyen que, al corregir el sesgo de selección, el apoyo público muestra efectos reales en las empresas beneficiarias (Aerts \& Schmidt, 2008; Carboni, 2011; Czarnitzki \& LopesBento, 2014; González \& Pazó, 2008; Hud \& Hussinger, 2015). En cuanto al tipo de actividades de innovación impulsadas por la financiación pública, las estimaciones muestran que, en promedio, las empresas privadas que reciben tratamiento son estimuladas a realizar ambos tipos; no obstante, la magnitud del efecto promedio del tratamiento es mayor en actividades de innovación exógena frente a las actividades endógenas. En el análisis sectorial, si bien se encuentran comportamientos análogos, los efectos son heterogéneos en su magnitud. Así, las empresas de ambos sectores son estimuladas a realizar actividades exógenas más que endógenas, pero con efectos mayores en las empresas manufactureras.

Acerca de si el financiamiento público aumenta la capacidad de las empresas a innovar en producto y/o proceso, los resultados ayudan a confirmar esta hipótesis. Sin embargo, se puede rescatar algunas diferencias entre periodos; durante el trienio 2009-2011 el apoyo público tiene un mayor impacto en la introducción de productos, mientras que lo contrario sucede en el periodo 2012-2014. A nivel sectorial, los resultados para las firmas manufactureras no difieren del resultado global. Para las empresas de servicios, el financiamiento público aumenta la capacidad para innovar en producto, pero no evidencia efectos sobre la capacidad de innovar en procesos.

Finalmente, se analiza si el apoyo público estimula la productividad de las empresas. Los resultados muestran que durante el periodo 2009-2011 la concesión de financiamiento público en innovación tiene efectos negativos sobre la productividad laboral; por el contrario, en el segundo periodo se evidencian efectos positivos y significativos. Por sectores, en manufactura se evidencia efectos positivos sobre el desempeño económico en el primer trienio, pero no hay efectos en el segundo periodo analizado; a diferencia del sector servicios, donde el efecto medio sobre la productividad laboral es negativo en el primer trienio, pero no muestra efectos en el segundo.

\section{DISCUSIÓN Y CONCLUSIONES}

Los resultados muestran que el financiamiento público es efectivo al estimular a las empresas ecuatorianas a invertir en innovación; una de las explicaciones posibles es que el apoyo fue otorgado a aquellas empresas que tienen experiencia en realizar actividades de innovación, pero que enfrentan problemas de financiamiento. Estos resultados son similares a los encontrados por Bukstein \& Usher (2016) y Crespi et al. (2011) en su estudio para América Latina. Sin embargo, las fuentes de financiamiento, ya sea a través de recursos propios, de la banca, del exterior o públicos, muestran ser un determinante fundamental para invertir en innovación. Por lo tanto, una sugerencia es que los organismos públicos competentes amplíen su financiamiento, no solo considerando firmas con experiencia en actividades innovadoras, sino también empresas sin experiencia en este tipo de actividades. Un resultado congruente con otros estudios (Lasarga et al., 2015) muestra que las empresas ecuatorianas realizan en mayor medida actividades de innovación exógena frente a la endógena, lo que significa que Ecuador se encuentra en el proceso de absorción de capacidades más que creación de las mismas.

Cabe señalar que el financiamiento público no solo incentiva a las empresas a invertir en actividades de innovación, sino también genera éxito en la introducción de un producto y/o proceso nuevo o significativamente mejorado. En el primer periodo analizado, las empresas beneficiadas mostraron interés en aumentar su productividad a largo plazo por medio de la innovación de productos, a diferencia del segundo periodo, donde las firmas se muestran más propensas a obtener resultados inmediatos sobre la productividad, mediante la innovación de procesos; esto explica las diferencias de efectos en el desempeño económico entre periodos. Así, el efecto negativo sobre la productividad laboral en el trienio 20092011 se puede deber a que las empresas beneficiadas propendieron a innovar en producto, con lo que el tiempo para obtener los rendimientos esperados es mayor. Por el contrario, los efectos positivos sobre la productividad laboral en el trienio 2012-2014 puede deberse a que las empresas tratadas se muestran más propensas a innovar en procesos, lo que permite obtener rendimientos en un menor plazo. Sin embargo, este resultado es en parte inesperado, debido a que parte de la literatura señala que es necesario más tiempo para observar el impacto sobre la productividad (Bukstein \& Usher, 2016; Czarnitzki \& Lopes-Bento, 2014).

Con respecto a los resultados a nivel sectorial, se confirma la hipótesis de efectos heterogéneos de la financiación pública; el impacto del apoyo público sobre la intensidad de innovar es mayor para las firmas manufactureras frente a las de servicios. Esto lleva a concluir que para las empresas del sector servicios no solo es importante disponer de financiamiento para realizar actividades de innovación, sino también otro tipo de factores, como disponer de mayores oportunidades tecnológicas. Por otro lado, solo existe evidencia de aumento en la productividad de las empresas beneficiadas del sector manufactura durante el primer trienio; de acuerdo a Aboal \& Garda (2015) este resultado puede deberse a que los costos de transacción para innovar en el sector servicios son más altos, debido a la intangibilidad de los servicios, razón por la que es más complicado proteger innovaciones en el sector servicios.

Es importante señalar que esta investigación enfrenta algunas limitaciones. En primer lugar, el periodo de análisis es muy corto, tiempo que no permite evidenciar efectos del financiamiento público a largo plazo, especialmente sobre la productividad. Por otro lado, no se dispone de información acerca del monto de la financiación, así como el año en el que las empresas se benefician del tratamiento. Tampoco se específica el tipo de financiación pública, lo que conlleva a suponer que el financiamiento es homogéneo para todas las empresas, un supuesto poco realista. Se debe tener en cuenta que el financiamiento se realiza con el fin de dar capacidades a las empresas para realizar actividades en innovación, pero los hallazgos de este estudio no permiten saber si una empresa que recibe financiamiento es impulsada a continuar invirtiendo e innovando, o solamente lo hace durante un periodo corto luego de ser financiada.

Finalmente, con el fin de mejorar los instrumentos de apoyo a la innovación es necesario estudiar la articulación entre los actores del Sistema Nacional de Innovación, puesto que para innovar no solo depende de actores como el gobierno y las empresas, sino de otros actores como las 
universidades y la sociedad.

\section{REFERENCIAS}

Aboal, D., \& Garda, P. (2015). ¿La financiación pública estimula la innovación y la productividad? Una evaluación de impacto. (Spanish). Revista de La CEPAL, 2015(115), 45-70.

Acs, Z. J., \& Audretsch, D. B. (1987). Innovation, market structure, and firm size. The Review of Economics and Statistics, 69(4), 567-574.

Aerts, K., \& Schmidt, T. (2008). Two for the price of one? On additionality effects of R \& D subsidies: A comparison between Flanders and Germany. Research Policy, 37(5), 806-822.

Aghion, P., David, P. A., \& Foray, D. (2009). Science, technology and innovation for economic growth: Linking policy research and practice in "STIG Systems." Research Policy, 38(4), 681-693. https://doi.org/10.1016/j.respol.2009.01.016

Arza, V., \& López, A. (2010). Innovation and productivity in the Argentine manufacturing sector (No. No. IDB-WP-187). IDB Working Paper Series (Vol. 187). https://doi.org/10.2139/ssrn.1817297

Audretsch, D. B., Coad, A., \& Segarra, A. (2014). Firm growth and innovation. Small Business Economics, 743-749. https://doi.org/10.1007/s11187-014-9560-x

Barona, B., Rivera, J. A., Aguilera, C. I., \& Marizado, P. A. (2015). Financiación de la innovación en Colombia. Entramado, 11(1), 80-93.

Benavente, J., Crespi, G., \& Maffioli, A. (2007). Public support to firm-level innovation: An evaluation of the Fontec Program. No 507, OVE Working Papers, Inter-American Development Bank, Office of Evaluation and Oversight (OVE).

Bukstein, D., \& Usher, X. (2016). Impactos de los instrumentos de promoción de la innovación empresarial. Documento de Discusión No. IDB-DP431.

Carboni, O. A. (2011). R\&D subsidies and private R\&D expenditures: Evidence from Italian manufacturing data. International Review of Applied Economics, 25(4), 419-439. https://doi.org/10.1080/02692171.2010.529427

Castro, L., \& Jorrat, D. (2013). Evaluación de impacto de programas públicos de financiamiento sobre la innovación y la productividad. El caso de los Servicios de Software e Informáticos de la Argentina. Documento de trabajo $\mathrm{N}^{\circ} 115$, CIPPEC.

Cimoli, M. (2010). Innovar para crecer: Desafios y oportunidades para el desarrollo sostenible e inclusivo en Iberoamerica. Santiago de Chile: Comision Economica para America Latina y el Caribe (CEPAL) / Secretaria General Iberoamericana (SEGIB).

Cerulli, G. (2011). ivtreatreg: a new STATA routine for estimating binary treatment models with heterogeneous response to treatment under observable and unobservable selection. CNR-Ceris Working Papers. Venice. https://doi.org/The Stata Journal
Chudnovsky, D., López, A., Rossi, M., \& Ubfal, D. (2006). Evaluating a program of public funding of private innovation activities. An econometric study of FONTAR in Argentina (OVE/WP-16/06). OVE Working Paper. Washington, D.C.

Clausen, T. H. (2009). Do subsidies have positive impacts on R\&D and innovation activities at the firm level? Structural Change and Economic Dynamics, 20(4), 239-253. https://doi.org/10.1016/j.strueco.2009.09.004

Crépon, B., Duguet, E., \& Mairessec, J. (1998). Research, innovation and productivity: An econometric analysis at the firm level. Economics of Innovation and New Technology, 115-158.

Crespi, G., Garone, L. F., Maffioli, A., \& Melendez, M. (2015). Long-term productivity effects of public support to innovation in Colombia. Emerging Markets Finance and Trade, 51(1), 48-64. https://doi.org/10.1080/1540496X.2015.998080

Crespi, G., Solís, G., \& Tacsir, E. (2011). Evaluación del impacto de corto plazo de SENACYT en la innovación de las Empresas Panameñas. Nota Técnica No. 263. Washington, D.C: BID.

Czarnitzki, D., \& Lopes-Bento, C. (2014). Innovation subsidies: Does the funding source matter for innovation intensity and performance? Empirical evidence from Germany. Industry and Innovation, 21(5), 380-409. https://doi.org/10.1080/13662716.2014.973246

Czarnitzki, D., \& Delanote, J. (2017). Incorporating innovation subsidies in the CDM framework: empirical evidence from Belgium. Economics of Innovation and New Technology, Taylor \& Francis Journals, 26(1-2), 78-92.

Dai, X., \& Cheng, L. (2015). The effect of public subsidies on corporate R\&D investment: An application of the generalized propensity score. Technological Forecasting and Social Change, 90(B), 410-419. https://doi.org/10.1016/j.techfore.2014.04.014

De Negri, J. A., Borges Lemos, M., \& De Negri, F. (2006). Impact of P\&D incentive program on the performance and technological efforts of Brazilian industrial firms (OVE/WP-14/06). OVE Working Paper. Washington, D.C.

Gertler, P., Martínez, S., Premand, P., Rawlings, L., Vermeerschm, C., Negocios, N. L. O. S., \& Municipales, E. (2009). La evaluación de impacto en la práctica. World Bank. https://doi.org/978-0-82138681-1

González, X., \& Pazó, C. (2008). Do public subsidies stimulate private R\&D spending? Research Policy, 37(3), 371-389. https://doi.org/10.1016/j.respol.2007.10.009

Greenhalgh, C., \& Rogers, M. (2009). The nature and importance of innovation. In: Innovation, Intellectual Property, and Economic Growth. New Jersey: Princeton University Press.

Guaipatin, C., \& Schwartz, L. (2014). Ecuador: Análisis del sistema nacional de innovación hacia la consolidación de una cultura innovadora. Washington D.C, BID. 
Guan, J., \& Pang, L. (2017). Industry specific effects on innovation performance in China. China Economic Review, 44, 125-137.

https://doi.org/10.1016/j.chieco.2017.03.013

Hall, B. (2010). The financing of innovative firms. Review of Economics and Institutions, 3880, 1-30. https://doi.org/10.5202/rei.v1i1.4

Hall, B., \& Lerner, J. (2009). The financing of R\&D and innovation. NBER Working Papers 15325, National Bureau of Economic Research, Inc.

Herrera, L. (2012). El efecto diferenciado de la financiación pública de la innovación: regiones centrales versus periféricas. Innovación y Competitividad ICE, (869), 81-98.

Hewitt-Dundas, N., \& Roper, S. (2010). Output additionality of public support for innovation: Evidence for Irish manufacturing plants. European Planning Studies, 18(1), 107-122. https://doi.org/10.1080/09654310903343559

Howell, A. J. (2017). Picking "winners" in China: Do subsidies matter for indigenous innovation and firm productivity? China Economic Review, 44, 154-165. https://doi.org/10.1016/j.chieco.2017.04.005

Hud, M., \& Hussinger, K. (2015). The impact of R\&D subsidies during the crisis. Research Policy, 44(10), 1844-1855. https://doi.org/10.1016/j.respol.2015.06.003

Huergo, E., Trenado, M., \& Ubierna, A. (2015). The impact of public support on firm propensity to engage in R\&D: Spanish experience. Technological Forecasting and Social Change, 113(B), 206-219. https://doi.org/10.1016/j.techfore.2015.05.011

INEC. (2011). Manual del encuestador del formulario de actividades de innovación. Periodo 2009-2011. Quito, Ecuador: Instituto Nacional de Estadística y Censos.

Jaramillo, Hernán Lugones, Gustavo Salazar, M. (2001). Normalización de indicadores de innovación tecnológica en América Latina y el Caribe. Manual de Bogotá. RICYT, OEA, CYTED, COLCIENCIASOCYT. Colciencias-OCYT.

Jiménez, L. F. (2008). Capital de riesgo e innovación en América Latina. CEPAL Review, 172-194.

Klette, T. J., Møen, J., \& Griliches, Z. (2000). Do subsidies to commercial R \& D reduce market failures? Microeconometric evaluation studies. Research Policy, 29(4-5), 471-495. https://doi.org/10.1016/S0048-7333(99)00086-4

Lasarga, E., Rosich, L., \& Rueda, H. (2015). Evaluación de impacto de las políticas de incentivo a la actividad innovadora en el sector industrial uruguayo. Series Documentos de Investigación Estudiantil No. DIE 02/2015 (Vol. 1963).

Lee, C. Y. (2011). The differential effects of public R\&D support on firm R\&D: Theory and evidence from multi-country data. Technovation, 31(5-6), 256-269. https://doi.org/10.1016/j.technovation.2011.01.006

López, A. (2009). Las evaluaciones de programas públicos de apoyo al fomento y desarrollo de la tecnología y la innovación en el sector productivo en América Latina: Una revisión crítica. Nota Técnica, Dialogo Regional de Política, Red de Innovación, Ciencia y Tecnología, BID.

Nooteboom, B., \& Stam, E. (Eds.). (2008). Microfoundations for innovation policy. Amsterdam: Amsterdam University Press. Retrieved from http://www.jstor.org/stable/j.ctt46mwvr.

OCDE-Eurosat. (2005). Manual de Oslo: Guía para la recogida einterpretación de datos sobre innovación. (OCDE) ( $3^{\mathrm{a}}$ ed.). https://doi.org/10.1787/9789264065659-es

ONUDI. (2016). Informe sobre el desarrollo industrial 2016. El rol de la tecnología y la innovación en el desarrollo industrial inclusivo y sostenible. $70 \mathrm{pp}$. Vienna, Autriche: ONUDI.

Radicic, D., \& Pugh, G. (2015). Input and output additionality of R\&D programmes in European SMEs. In European Conference on Innovation and Entrepreneurship (p. 567).

RICYT. (2016). Indicadores. Retrieved August 8, 2017 , from http://www.ricyt.org/indicadores

SENPLADES. (2013). Plan nacional buen vivir. Quito, Ecuador: Secretaría Nacional de Desarrollo y Planificación.

Szczygielski, K., Grabowski, W., Pamukcu, M. T., \& Tandogan, V. S. (2016). Does government support for private innovation matter? Firm-level evidence from two catching-up countries. Research Policy, 46(1), 219-237. https://doi.org/10.1016/j.respol.2016.10.009

Vila, M., Ferro, C., \& Guisado, M. (2009). Innovación, financiación pública y tamaño empresarial. Cuadernos de Gestión, 10, 75-88.

Wooldridge, J. M. (2002). Econometric analysis of cross section and panel data (Vol. 58). London UK: The MIT Press. https://doi.org/10.1515/humr.2003.021

Wooldridge, J. M. (2009). Introducción a la econometría. Un enfoque moderno. 890 pp. Cengage Learning (Eds.) ( $4^{\mathrm{a}}$ ed.).

Zúñiga, P., \& Crespi, G. (2013). Innovation strategies and employment in Latin American firms. Structural Change and Economic Dynamics, 24, 1-17. https://doi.org/10.1016/j.strueco.2012.11.001 
Anexo 1. Medición y signo esperado de variables

Tabla 3. Variables modelo probit.

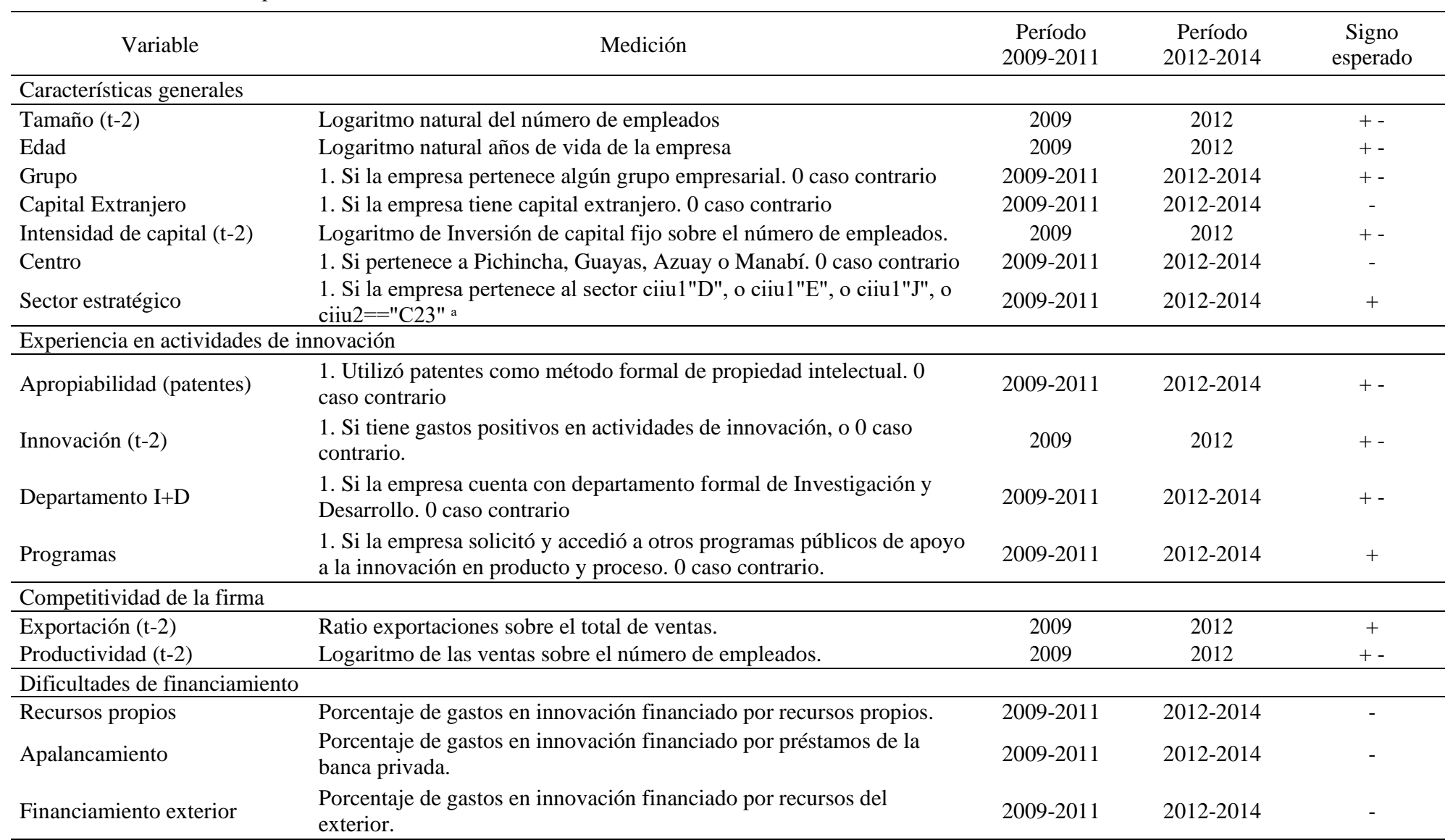

a ciiu1"D" suministro de electricidad, gas, vapor y aire acondicionado; ciiu1"E" distribución de agua; alcantarillado, gestión de desechos y actividades de saneamiento; ciiu1"J" información y comunicación; ciiu2"C23" fabricación de otros productos minerales no metálicos

Fuente: Elaboración propia sobre la base de datos de la Encuesta Nacional de Innovación (ENAI) de los periodos 2009-2011 y 2012-2014 del

Instituto Nacional de Estadísticas y Censos (INEC). 
Tabla 4. Variables independientes utilizadas, hipótesis 1-2-3.

\begin{tabular}{|c|c|c|c|c|}
\hline Variable & Medición & $\begin{array}{c}\text { Período } \\
2009-2011\end{array}$ & $\begin{array}{c}\text { Período } \\
2012-2014\end{array}$ & $\begin{array}{l}\text { Signo } \\
\text { esperado }\end{array}$ \\
\hline Variables dependientes & \multicolumn{4}{|c|}{$\begin{array}{l}\text { Inversión total, Inversión privada (Hipótesis uno) Actividades endógenas y exógenas (Hipótesis dos) } \\
\text { Innovación en productos, Innovación en procesos (Hipótesis tres) }\end{array}$} \\
\hline \multicolumn{5}{|c|}{ Tratamiento } \\
\hline Apoyo gubernamental predicho & $\begin{array}{l}\text { Probabilidad de recibir financiamiento público en innovación } \\
\text { estimado }\end{array}$ & & & +- \\
\hline \multicolumn{5}{|l|}{ Características generales } \\
\hline Tamaño & Logaritmo natural del número de empleados & 2011 & 2014 & +- \\
\hline Edad & Logaritmo natural años de vida de la empresa & 2009 & 2012 & +- \\
\hline Grupo & $\begin{array}{l}\text { 1. Si la empresa pertenece algún grupo empresarial. } 0 \text { caso } \\
\text { contrario }\end{array}$ & 2009-2011 & 2012-2014 & +- \\
\hline Capital Extranjero & 1. Si la empresa tiene capital extranjero. 0 caso contrario & 2009-2011 & 2012-2014 & +- \\
\hline Intensidad de capital & $\begin{array}{l}\text { Logaritmo de Inversión de capital fijo sobre el número de } \\
\text { empleados. }\end{array}$ & 2011 & 2014 & +- \\
\hline Centro & $\begin{array}{l}\text { 1. Si pertenece a Pichincha, Guayas, Azuay o Manabí. } 0 \text { caso } \\
\text { contrario }\end{array}$ & 2009-2011 & 2012-2014 & +- \\
\hline $\begin{array}{l}\text { Sector servicio media alta } \\
\text { tecnología }\end{array}$ & $\begin{array}{l}\text { 1. Si la empresa pertenece al sector servicio de media alta } \\
\text { tecnología. } 0 \text { caso contrario. }\end{array}$ & 2009-2011 & 2012-2014 & +- \\
\hline $\begin{array}{l}\text { Sector manufactura media alta } \\
\text { tecnología }\end{array}$ & $\begin{array}{l}\text { 1. Si la empresa pertenece al sector de manufactura de media alta } \\
\text { tecnología. } 0 \text { caso contrario. }\end{array}$ & 2009-2011 & 2012-2014 & +- \\
\hline Sector a nivel de letra & Sector código CIIU a nivel de letra & & & \\
\hline \multicolumn{5}{|c|}{ Experiencia en actividades de innovación } \\
\hline Apropiabilidad (patentes) & $\begin{array}{l}\text { 1. Utilizó patentes como método formal de propiedad intelectual. } 0 \\
\text { caso contrario }\end{array}$ & $2009-2011$ & 2012-2014 & +- \\
\hline $\mathrm{I}+\mathrm{D}(\mathrm{t}-2)$ & $\begin{array}{l}\text { Gastos positivos en actividades de innovación sobre las ventas } \\
\text { totales }\end{array}$ & 2009 & 2012 & +- \\
\hline Departamento I+D & $\begin{array}{l}\text { 1. Si la empresa cuenta con departamento formal de Investigación y } \\
\text { Desarrollo. }\end{array}$ & 2009-2011 & $2012-2014$ & +- \\
\hline Programas & $\begin{array}{l}1 \text { si la empresa solicitó y accedió a otros programas públicos de } \\
\text { apoyo a la innovación en producto y proceso. } 0 \text { caso contrario. }\end{array}$ & $2009-2011$ & 2012-2014 & + \\
\hline \multicolumn{5}{|l|}{ Competitividad de la firma } \\
\hline Exportación (t-2) & Monto total de exportaciones sobre las ventas totales & 2009 & 2012 & + \\
\hline Productividad $(\mathrm{t}-2)$ & Log de las ventas sobre el número de empleados. & 2009 & 2012 & +- \\
\hline \multicolumn{5}{|l|}{ Dificultades de financiamiento } \\
\hline Recursos propios & $\begin{array}{l}\text { Porcentaje de gastos en innovación financiado por recursos propios. } \\
0 \text { caso contrario. }\end{array}$ & $2009-2011$ & $2012-2014$ & + \\
\hline Apalancamiento & $\begin{array}{l}\text { Porcentaje de gastos en innovación financiado por préstamos de la } \\
\text { banca privada. } 0 \text { caso contrario. }\end{array}$ & $2009-2011$ & 2012-2014 & + \\
\hline
\end{tabular}

MASKANA, Vol. 11, No. 1, 33-47, 2020

doi: $10.18537 / \mathrm{mskn} .11 .01 .04$ 


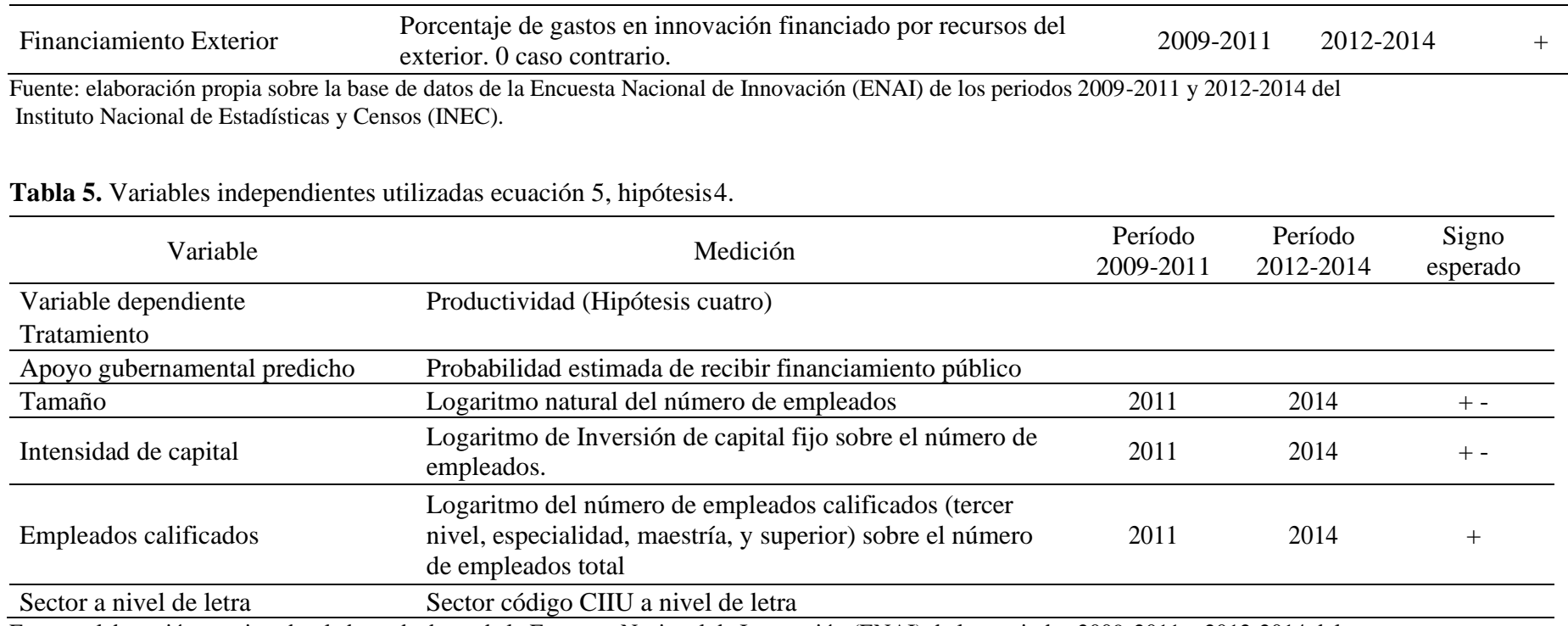

Fuente: elaboración propia sobre la base de datos de la Encuesta Nacional de Innovación (ENAI) de los periodos 2009-2011 y 2012-2014 del

Instituto Nacional de Estadísticas y Censos (INEC). 
Anexo 2.

Tabla 6. Efectos marginales modelo probit.

\begin{tabular}{|c|c|c|c|c|c|c|c|c|c|c|c|c|c|c|c|c|c|c|}
\hline \multirow{4}{*}{$\begin{array}{l}\text { Variable dependiente (Financiación } \\
\text { pública) }\end{array}$} & \multicolumn{6}{|c|}{ Todas las empresas } & \multicolumn{6}{|c|}{ Empresas manufactureras } & \multicolumn{6}{|c|}{ Empresas servicios } \\
\hline & \multicolumn{3}{|c|}{$2009-2011$} & \multicolumn{3}{|c|}{$2012-2014$} & \multicolumn{3}{|c|}{$2009-2011$} & \multicolumn{3}{|c|}{$2012-2014$} & \multicolumn{3}{|c|}{$2009-2011$} & \multicolumn{3}{|c|}{$2012-2014$} \\
\hline & \multicolumn{2}{|c|}{$\begin{array}{c}\text { Efectos } \\
\text { marginales }\end{array}$} & \multirow[t]{2}{*}{ Std. Err. } & \multicolumn{2}{|c|}{$\begin{array}{c}\text { Efectos } \\
\text { marginales }\end{array}$} & \multirow[t]{2}{*}{ Std. Err. } & \multicolumn{2}{|c|}{$\begin{array}{c}\text { Efectos } \\
\text { marginales }\end{array}$} & \multirow[t]{2}{*}{ Std. Err. } & \multicolumn{2}{|c|}{$\begin{array}{c}\text { Efectos } \\
\text { marginales }\end{array}$} & \multirow[t]{2}{*}{ Std. Err. } & \multicolumn{2}{|c|}{$\begin{array}{c}\text { Efectos } \\
\text { marginales }\end{array}$} & \multirow[t]{2}{*}{ Std. Err. } & \multicolumn{2}{|c|}{$\begin{array}{c}\text { Efectos } \\
\text { marginales }\end{array}$} & \multirow[t]{2}{*}{ Std. Err. } \\
\hline & & & & & & & & & & & & & & & & & & \\
\hline Tamaño (t-2) & -0.004 & & $(0.0027)$ & 0.0016 & & $(0.0016)$ & -0.002 & & $(0.0039)$ & 0.0007 & & $(0.0037)$ & -0.001 & & $(0.0064)$ & 0.0014 & & $(0.0059)$ \\
\hline Edad & 0.0105 & $* * *$ & $(0.0038)$ & 0.001 & & $(0.0025)$ & 0.0047 & & $(0.0050)$ & 0.0062 & & $(0.0048)$ & 0.0075 & & $(0.0083)$ & 0.0066 & & $(0.0069)$ \\
\hline Grupo & -0.004 & & $(0.0101)$ & -0.008 & & $(0.0059)$ & -0.017 & & $(0.0126)$ & -0.024 & $*$ & $(0.0121)$ & -0.028 & & $(0.0195)$ & -0.036 & ** & $(0.0177)$ \\
\hline Capital Extranjero & -0.015 & & $(0.0183)$ & -0.011 & & $(0.0076)$ & 0.0044 & & $(0.0159)$ & -0.012 & & $(0.0154)$ & 0.004 & & $(0.0249)$ & -0.011 & & $(0.0218)$ \\
\hline Intensidad de capital & 0.0008 & & $(0.0010)$ & 0.0007 & & $(0.0007)$ & 0.001 & & $(0.0010)$ & 0.0011 & & $(0.0013)$ & 0.0014 & & $(0.0017)$ & 0.0004 & & $(0.0021)$ \\
\hline Centro & -0.009 & & $(0.0063)$ & -0.002 & & $(0.0048)$ & -0.009 & & $(0.0084)$ & 0.0035 & & $(0.0093)$ & -0.013 & & $(0.0141)$ & 0.0108 & & $(0.0143)$ \\
\hline Sector estratégico & 0.0149 & $* *$ & $(0.0072)$ & 0.0031 & & $(0.0068)$ & 0.0221 & * & $(0.0133)$ & 0.0064 & & $(0.0157)$ & 0.0323 & & $(0.0231)$ & 0.0061 & & $(0.0233)$ \\
\hline \multicolumn{19}{|c|}{ Experiencia en actividades de innovación } \\
\hline Apropiabilidad (patentes) & 0.0103 & & $(0.0083)$ & 0.0087 & & $(0.0063)$ & -0.004 & & $(0.0101)$ & 0.0045 & & $(0.0116)$ & -0.008 & & $(0.0156)$ & $-6 \mathrm{E}-04$ & & $(0.0139)$ \\
\hline Innovación (t-2) & 0.0542 & $* * *$ & $(0.0178)$ & 0.0571 & $* * *$ & $(0.0145)$ & 0.0898 & $* * *$ & $(0.0320)$ & 0.0811 & $* * *$ & $(0.0273)$ & 0.0927 & $* *$ & $(0.0411)$ & 0.0459 & $* *$ & $(0.0216)$ \\
\hline Departamento I+D & -0.001 & & $(0.0075)$ & 0.003 & & $(0.0064)$ & -0.002 & & $(0.0093)$ & 0.0056 & & $(0.0115)$ & -0.004 & & $(0.0142)$ & 0.0107 & & $(0.0152)$ \\
\hline Programas & 0.0212 & $* * *$ & $(0.0069)$ & 0.0195 & $* * *$ & $(0.0045)$ & 0.0207 & $* *$ & $(0.0098)$ & 0.0244 & $* * *$ & $(0.0082)$ & 0.028 & $* *$ & $(0.0142)$ & 0.0176 & & $(0.0116)$ \\
\hline \multicolumn{19}{|l|}{ Competitividad de la firma } \\
\hline Exportación (t-2) & 0.0057 & & $(0.0128)$ & 0.0034 & & $(0.0109)$ & -0.003 & & $(0.0166)$ & 0.002 & & $(0.0173)$ & 0.0026 & & $(0.0258)$ & -0.002 & & $(0.0255)$ \\
\hline Productividad $(\mathrm{t}-2)$ & -0.004 & $*$ & $(0.0021)$ & 0.0024 & & $(0.0016)$ & 0.0033 & & $(0.0031)$ & 0.0008 & & $(0.0033)$ & 0.0064 & & $(0.0043)$ & 0.0019 & & $(0.0052)$ \\
\hline \multicolumn{19}{|l|}{ Dificultades de financiamiento } \\
\hline Recursos propios & -0.051 & $* * *$ & $(0.0161)$ & -0.061 & $* * *$ & $(0.0144)$ & -0.082 & $* * *$ & $(0.0286)$ & -0.093 & $* * *$ & $(0.0263)$ & -0.145 & $* * *$ & $(0.0356)$ & -0.175 & $* * *$ & $(0.0227)$ \\
\hline Apalancamiento & -0.027 & & $(0.0195)$ & -0.046 & $* * *$ & $(0.0150)$ & -0.070 & $* *$ & $(0.0305)$ & -0.065 & $* *$ & $(0.0261)$ & -0.128 & $* * *$ & $(0.0362)$ & -0.148 & $* * *$ & $(0.0207)$ \\
\hline Financiamiento Exterior & -0.002 & & $(0.0288)$ & -0.025 & & $(0.0189)$ & & & & -0.039 & & $(0.0339)$ & & & & -0.109 & $* * *$ & $(0.0353)$ \\
\hline N. observaciones & 2420 & & & 3873 & & & 1141 & & & 1576 & & & 688 & & & 922 & & \\
\hline Pseudo R2 & 0.1953 & & & 0.1916 & & & 0.254 & & & 0.1785 & & & 0.2691 & & & 0.3461 & & \\
\hline Wald chi & 109.1 & & & 99.55 & & & 60.41 & & & 45.78 & & & 54.62 & & & 112.26 & & \\
\hline Log likelihood & -183.4 & & & -273.4 & & & -78.11 & & & -165 & & & -68.67 & & & -115.61 & & \\
\hline
\end{tabular}
$-183.4$ $-273$.

Fuente: elaboración propia sobre la base de datos de la Encuesta Nacional de Innovación (ENAI) de los periodos 2009-2011 y 2012-2014 del Instituto Nacional de Estadísticas y Censos (INEC). 
Anexo 3.

Tabla 7. Efecto medio del financiamiento público por sector.

\begin{tabular}{|c|c|c|c|c|c|c|c|c|}
\hline \multirow{3}{*}{ Hipótesis } & \multicolumn{4}{|c|}{ Manufactura } & \multicolumn{4}{|c|}{ Servicios } \\
\hline & \multirow{2}{*}{\multicolumn{2}{|c|}{$\begin{array}{l}\text { 2009-2011 } \\
\text { ATE }\end{array}$}} & \multirow{2}{*}{\multicolumn{2}{|c|}{$\begin{array}{l}\text { 2012-2014 } \\
\text { ATE }\end{array}$}} & \multirow{2}{*}{\multicolumn{2}{|c|}{$\begin{array}{l}\text { 2009-2011 } \\
\text { ATE }\end{array}$}} & \multirow{2}{*}{\multicolumn{2}{|c|}{$\begin{array}{l}\text { 2012-2014 } \\
\text { ATE }\end{array}$}} \\
\hline & & & & & & & & \\
\hline \multicolumn{9}{|c|}{ Parte I - Hipótesis 1: Efectos de la financiación pública en los inputs } \\
\hline \multirow[t]{2}{*}{ Inversión total } & 12.91 & $* * *$ & 10.20 & $* * *$ & 5.89 & $* * *$ & 7.33 & $* * *$ \\
\hline & $(1.3307)$ & & $(1.4957)$ & & $(1.1898)$ & & $(2.1694)$ & \\
\hline \multirow[t]{2}{*}{ Inversión privada } & 13.44 & $* * *$ & 8.74 & $* * *$ & 6.23 & $* * *$ & 4.08 & $* *$ \\
\hline & $(0.9768)$ & & $(1.9503)$ & & $(1.2907)$ & & $(1.9236)$ & \\
\hline \multicolumn{9}{|c|}{ Parte II - Hipótesis 2: Efectos en el tipo de actividades de innovación } \\
\hline \multirow[t]{2}{*}{ Actividades endógenas } & 7.82 & $* * *$ & 6.62 & $* * *$ & 3.52 & $* * *$ & 3.59 & $* * *$ \\
\hline & $(1.1956)$ & & $(1.2477)$ & & $(0,9130)$ & & $(1.3529)$ & \\
\hline \multirow[t]{2}{*}{ Actividades exógenas } & 10.05 & $* * *$ & 9.28 & $* * *$ & 5.40 & $* * *$ & 7.28 & $* * *$ \\
\hline & $(1.8382)$ & & $(1.4971)$ & & $(0.9482)$ & & $(2.0981)$ & \\
\hline \multicolumn{9}{|c|}{ Parte III - Hipótesis 3: Efectos sobre la capacidad de innovar } \\
\hline \multirow[t]{2}{*}{ Innovación en productos } & 1.31 & $* * *$ & 1.4 & $* * *$ & 1.44 & $* * *$ & 1.59 & $* * *$ \\
\hline & $(0.2664)$ & & $(0.2173)$ & & $(0.4723)$ & & $(0.3174)$ & \\
\hline \multirow[t]{2}{*}{ Innovación en procesos } & 1.08 & $* * *$ & 1.75 & $* * *$ & 0.00 & & 1.32 & $* * *$ \\
\hline & $(0.2120)$ & & $(0.2082)$ & & $(0.1766)$ & & $(0.2941)$ & \\
\hline
\end{tabular}

Parte IV - Hipótesis 4: Efectos del financiamiento público en el desempeño de las empresas

\begin{tabular}{|c|c|c|c|c|c|}
\hline Productividad & $\begin{array}{r}1.80 \\
(1.0194)\end{array}$ & $\begin{array}{r}-0.0306 \\
(0.4703)\end{array}$ & $\begin{array}{r}-5.70 \\
(0.5357)\end{array}$ & $* * *$ & $\begin{array}{r}1.45 \\
(1.0316)\end{array}$ \\
\hline $\mathrm{N}^{\circ}$ Observaciones & 1155 & 1571 & 1265 & & 2290 \\
\hline
\end{tabular}

Nota: errores estándar robustos entre paréntesis. *** $\mathrm{p}<0.01 ; * * \mathrm{p}<0.05 ; * \mathrm{p}<0.1$.

Fuente: elaboración propia sobre la base de datos de la Encuesta Nacional de Innovación (ENAI) de los periodos 20092011 y 2012-2014 del Instituto Nacional de Estadísticas y Censos (INEC). 